Supporting Information for:

\title{
Non-Uniform Sampling in NMR Spectroscopy and the Preservation of Spectral Knowledge in the Time and Frequency Domains
}

\author{
Manpreet Kaur, ${ }^{1}$ Callie M. Lewis, ${ }^{1}$ Aaron Chronister, ${ }^{1,2}$ \\ Gabriel S. Phun, ${ }^{1,3}$ and Leonard J. Mueller ${ }^{1, *}$
}

${ }^{1}$ Department of Chemistry, University of California, Riverside, 92521

${ }^{2}$ Current address: Department of Physics and Astronomy, University of California, Los Angeles, 90095

${ }^{3}$ Current address: Department of Chemistry, University of California, Irvine, 92697

* corresponding author: leonard.mueller@ucr.edu; ORCID ID: 0000-0002-2607-9875 


\section{Table of Contents}

Figure S1: Construction of time-domain NUWS, Apodization-Weighted, and NUWS-UCR FID's

Figure S2: Convergence of Monte Carlo simulations ………....................................S4

Figure S3: NUS knowledge enhancements for two peaks with equal linewidths .....S5

Figure S4: NUS knowledge enhancements for two peaks with different linewidths ..S6

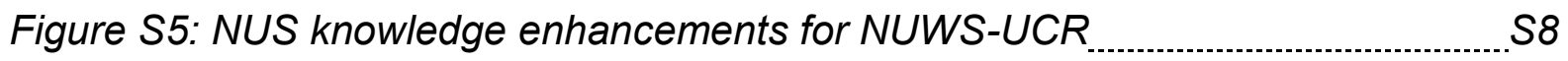

Figure S6: NUS knowledge enhancements NUWS-UCR and BFT-UCR for two peaks S9

Figure S7: NUS knowledge enhancements for BFT-UCR. S10 
a) NUS - TD
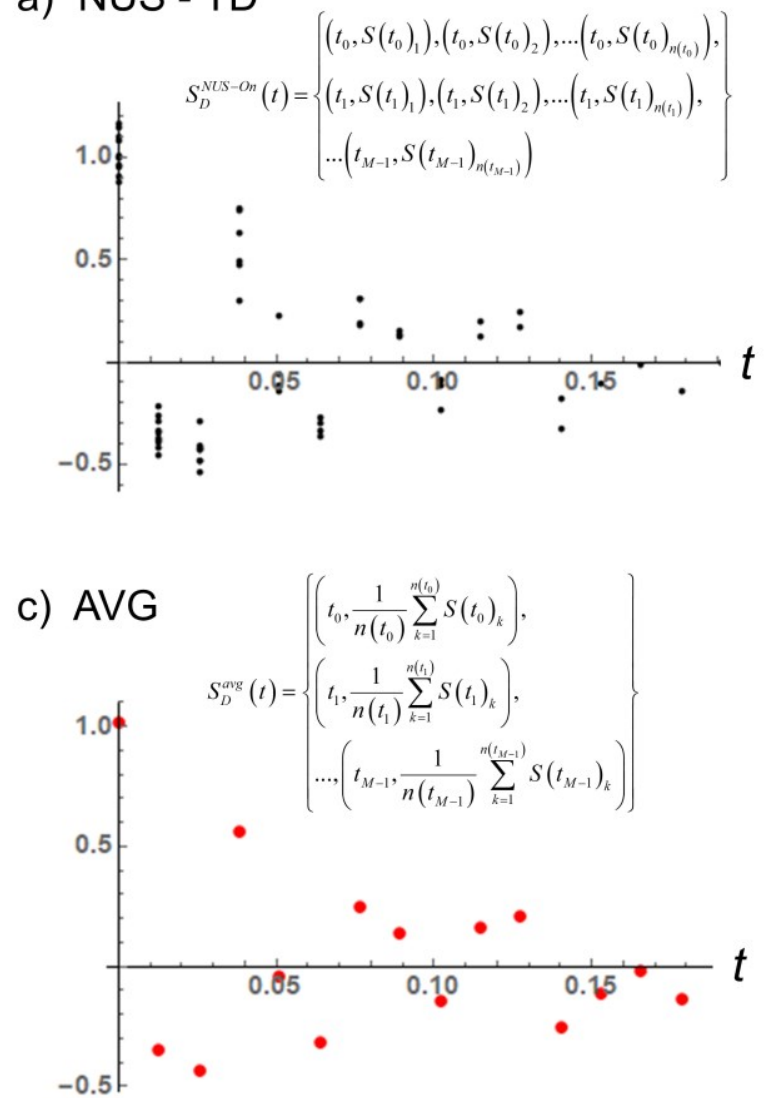

b) NUWS
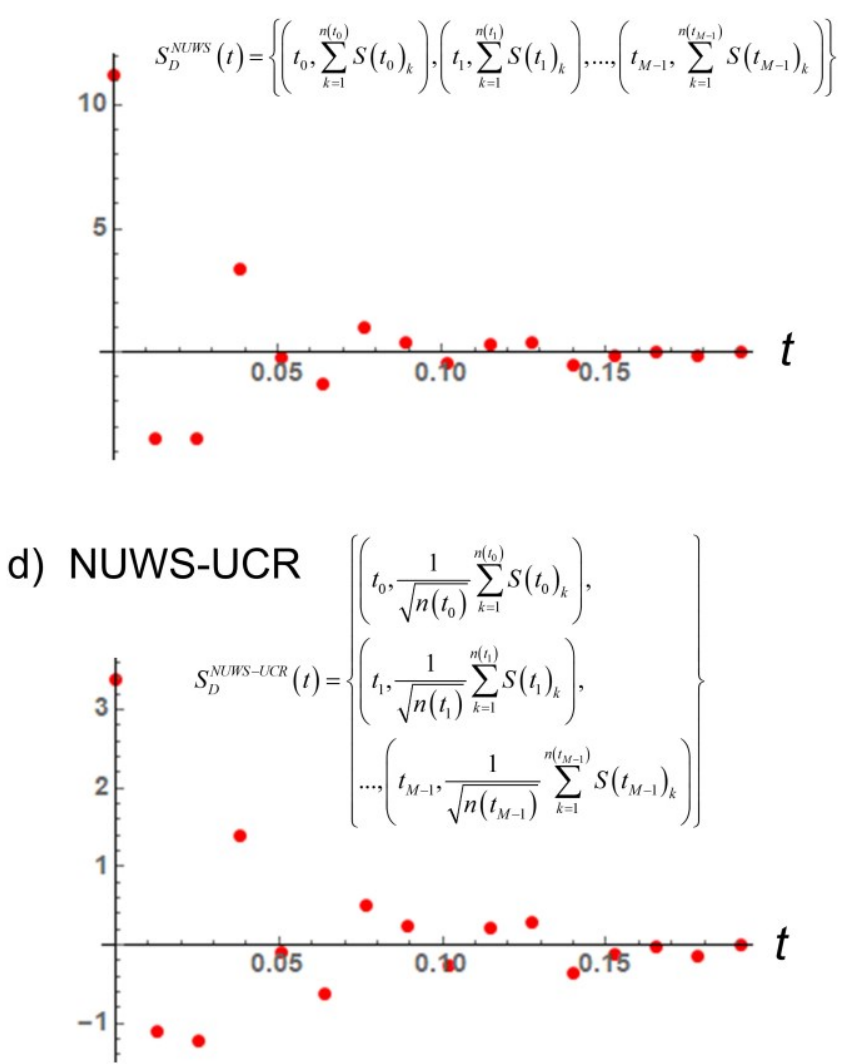

Figure S1: Construction of the (b) NUWS, (c) apodization-weighted/average, and (d) NUWS-UCR timedomain FID's from the (a) original NUS time-domain data. 

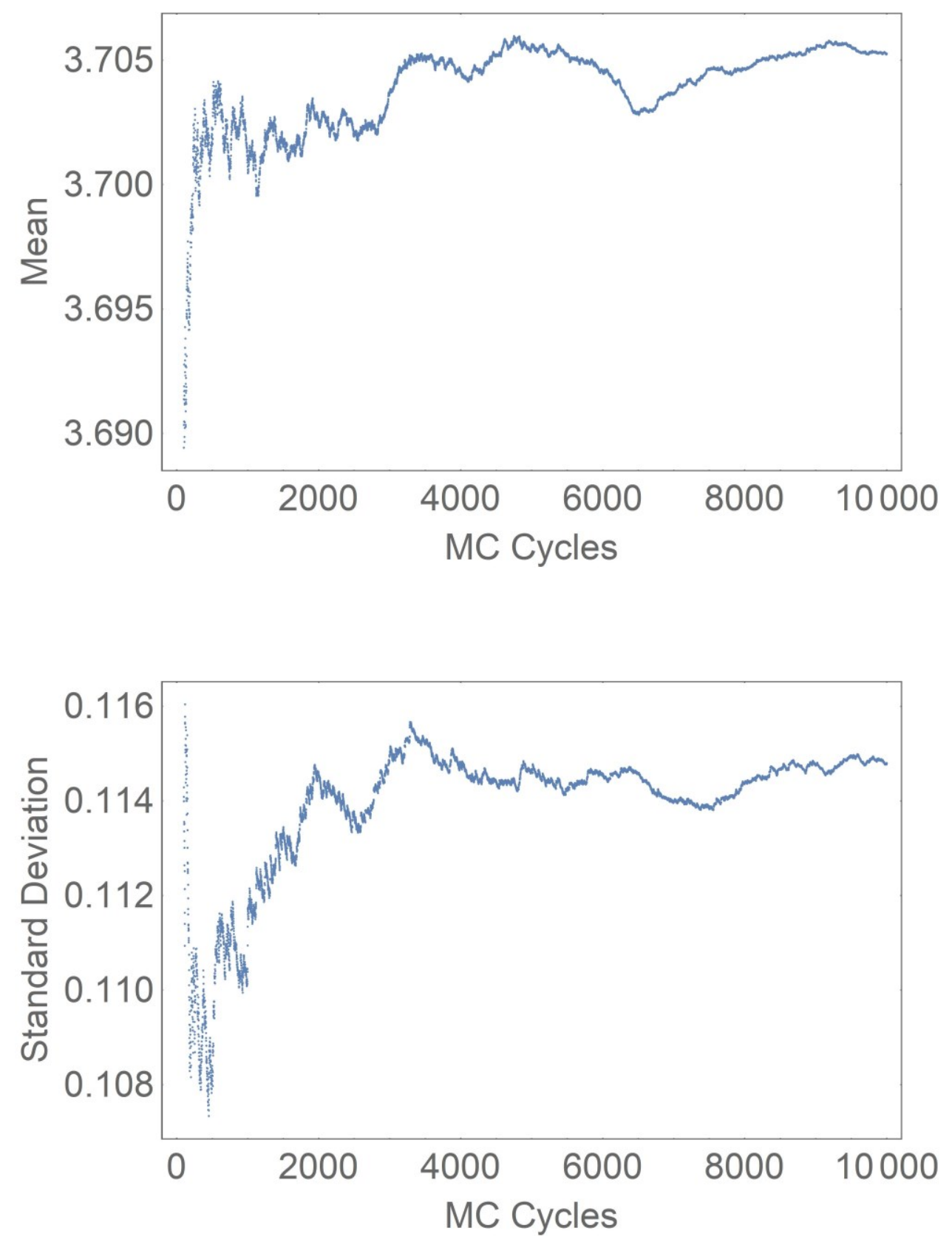

Figure S2: Example convergence of the mean and standard deviation of the peak area as a function of Monte Carlo cycles. 
a) 5 widths

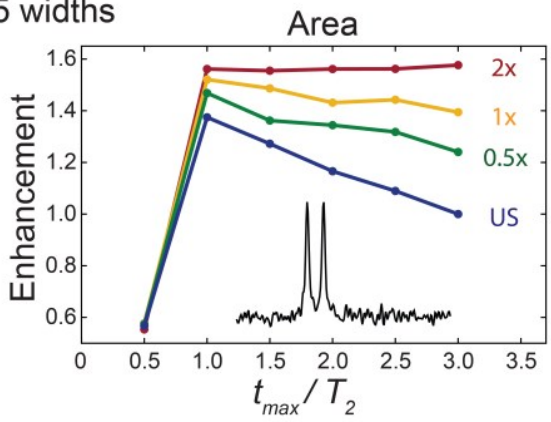

b) 2 widths

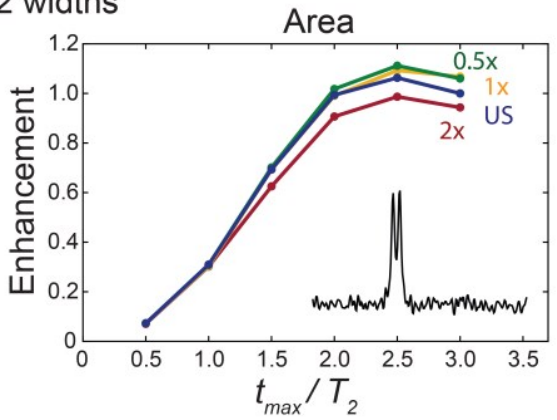

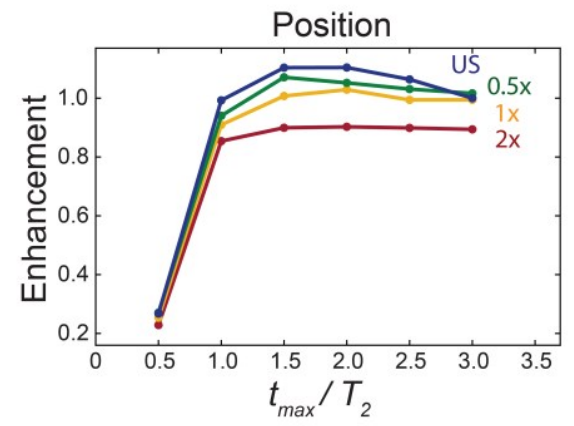
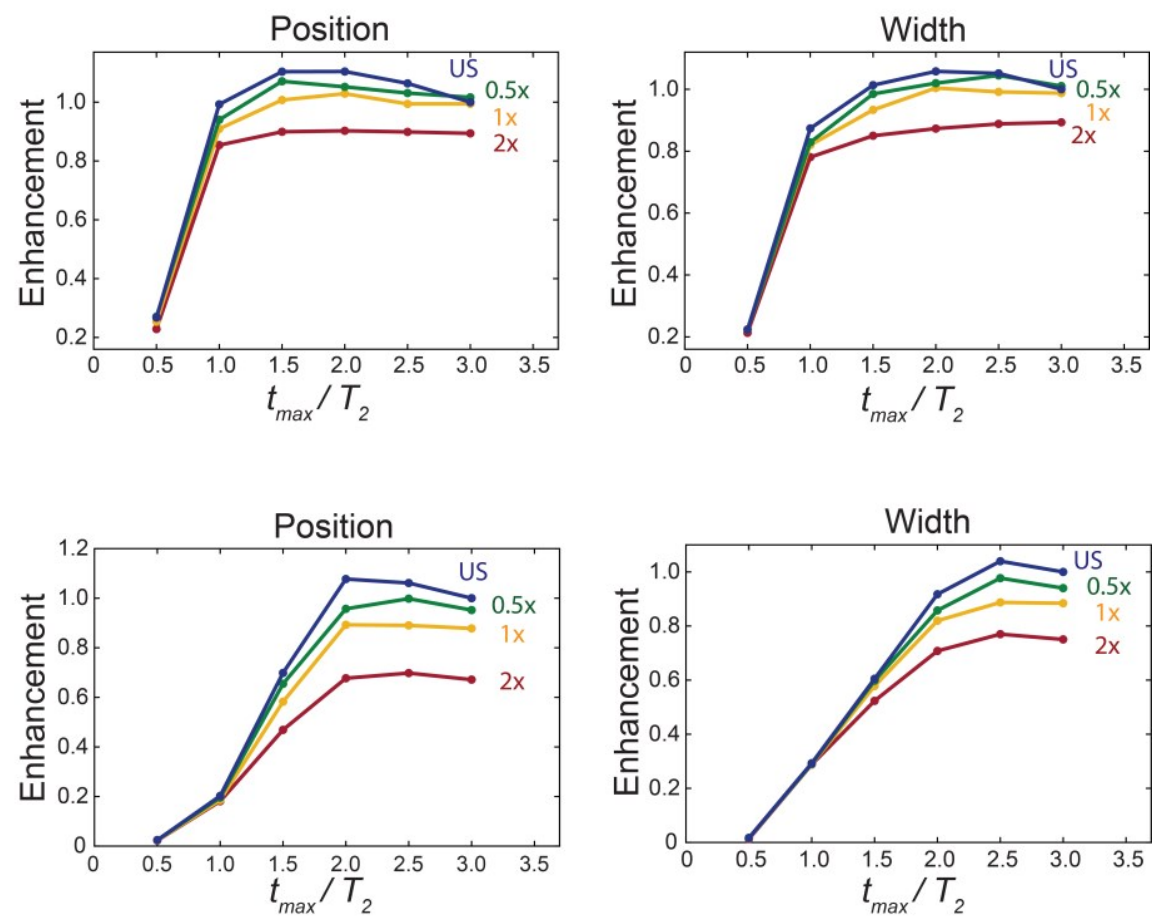

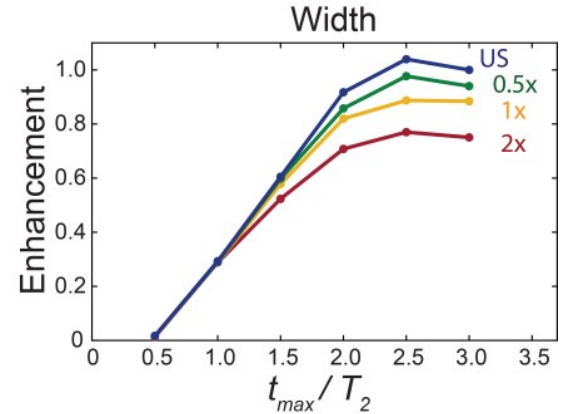

Figure S3: NUS knowledge enhancements in peak amplitude (area), frequency offset (position), and linewidth for one of two peaks with equal amplitude and linewidth separated by (a) 5 or (b) 2 times the linewidth under the indicated NUS biases. For each simulation, the total number of time-domain points sampled was held constant, corresponding to equivalent total experiment times. When the lines are well-separated, the enhancements mirror those for a single peak. As the lines merge, much of the NUS advantage is lost, although less aggressive biases continue to outperform US even in this limit. In all cases, the intrinsic spectral knowledge was extracted from the time-domain FID's; reference spectra were generated from US to $3 T_{2}$. 
a)
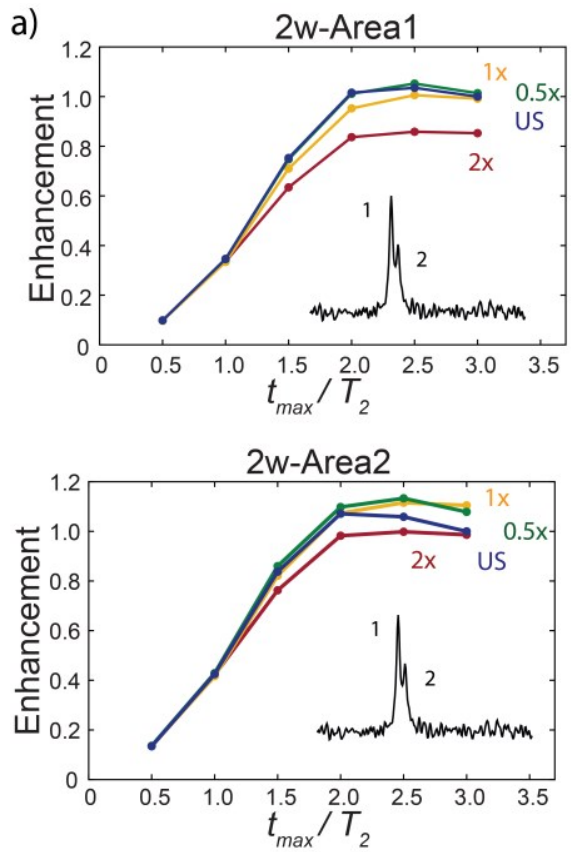

b)

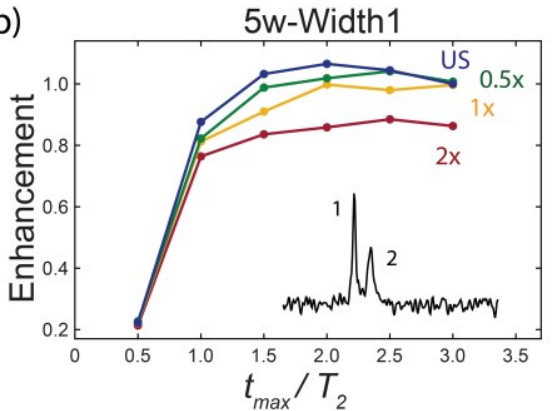

$5 \mathrm{w}-$ Area2

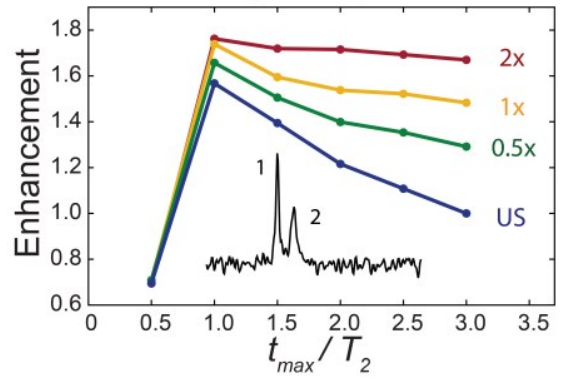

2w-Pos1
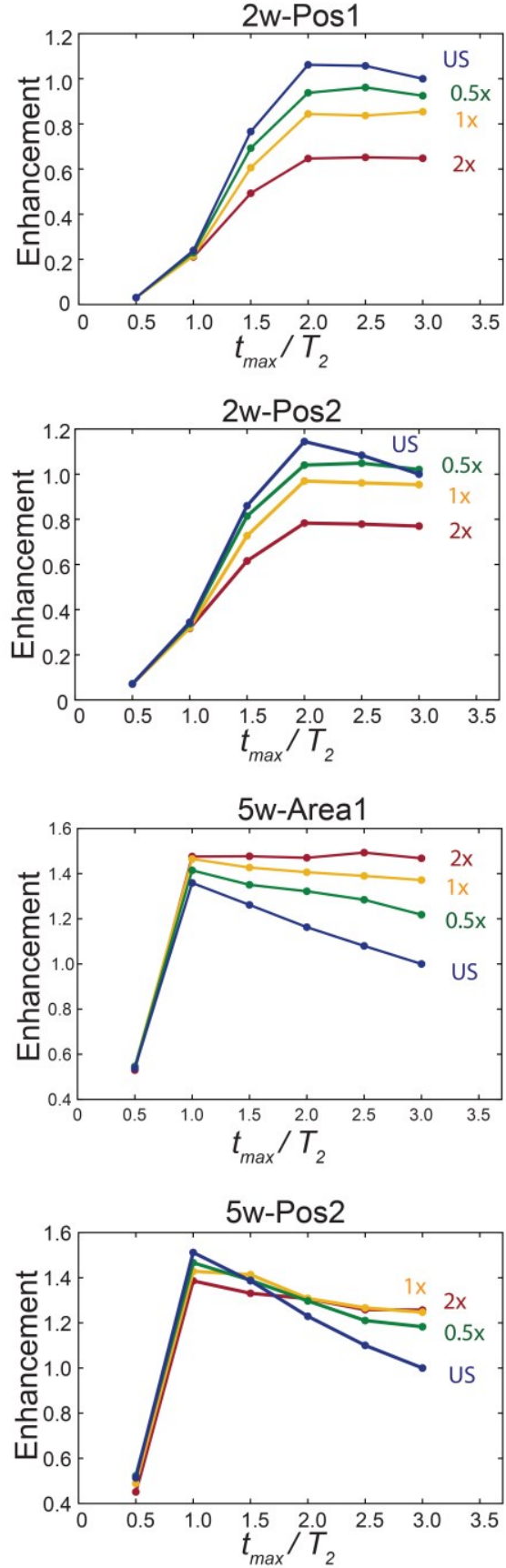

2w-Width1
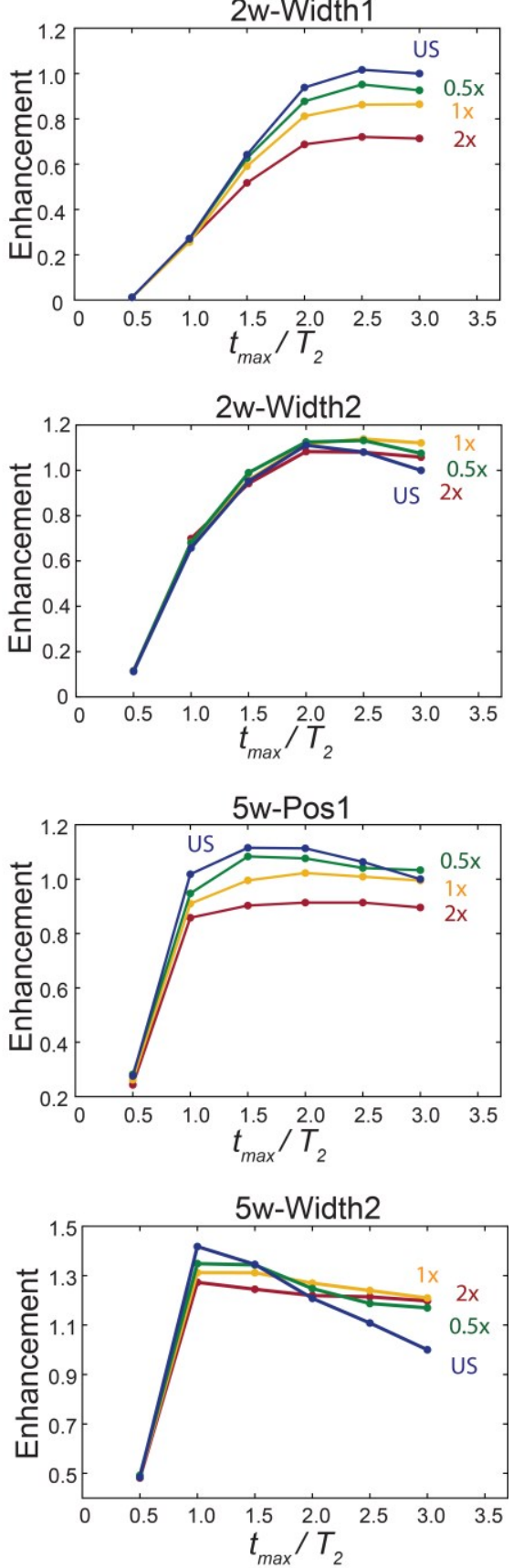

Figure S4: NUS knowledge enhancements in peak amplitude (area), frequency offset (position), and linewidth for two peaks separated by (a) 2 or (b) 5 times the linewidth under the indicated NUS biases. The peaks have equal amplitudes, but the second peak has a linewidth twice that of the first. Both the separation and the NUS biases are defined with respect to the linewidth of peak 1 . For each simulation, the total number of time-domain points sampled was held constant, corresponding to equivalent total experiment times. In all cases, the intrinsic spectral knowledge was extracted from the time-domain FID's; reference spectra were generated from US to $3 T_{2}$. 
c)

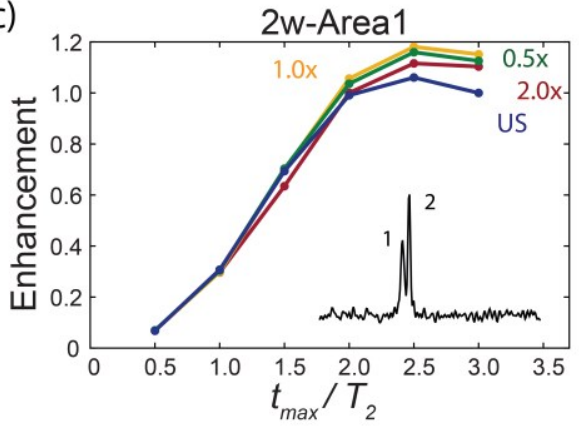

2w-Area2

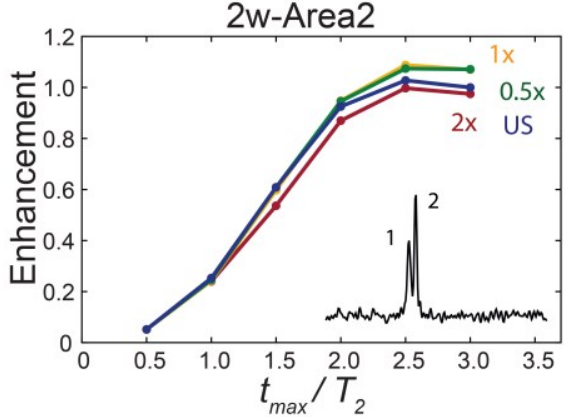

d)

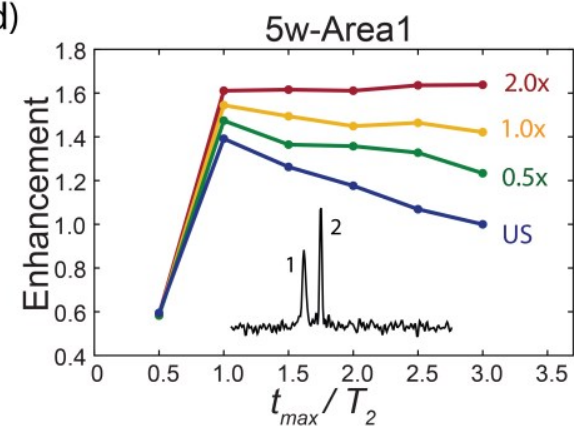

5 w-Area2

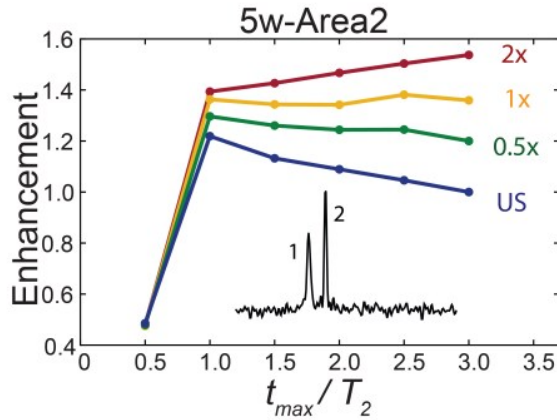

$2 \mathrm{w}-\mathrm{Pos} 1$

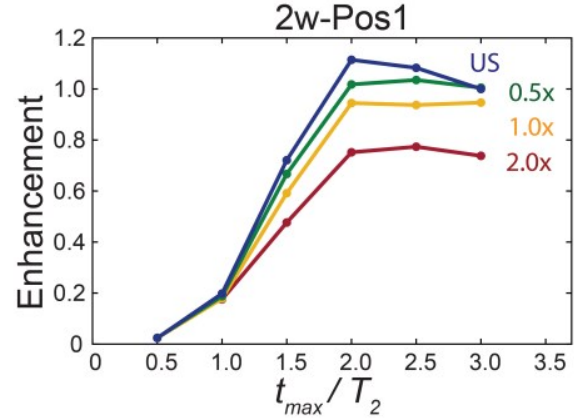

$2 \mathrm{w}-\mathrm{Pos} 2$

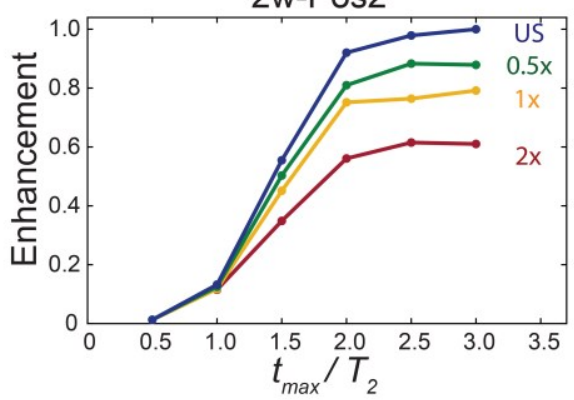

5w-Pos1

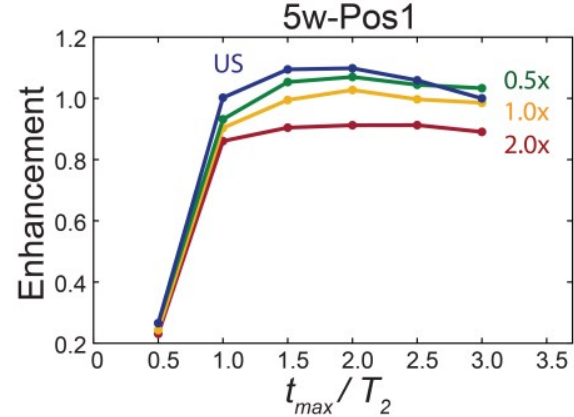

5w-Pos2

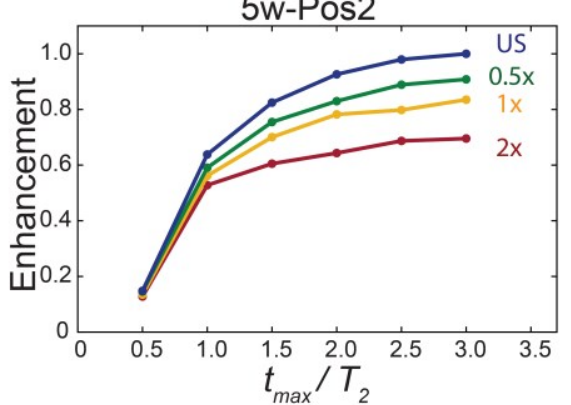

2w-Width1

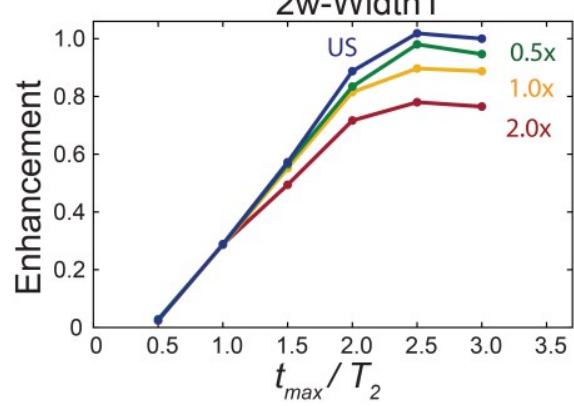

2w-Width2

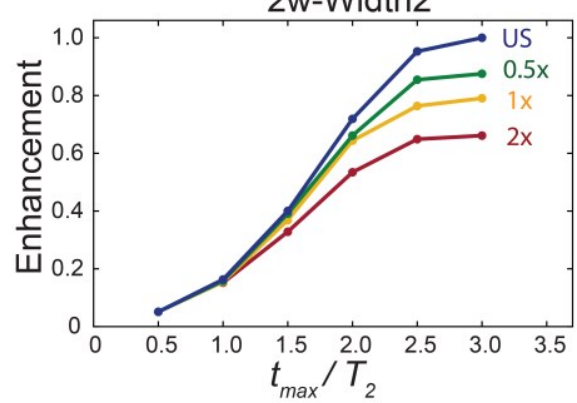

5w-Width1

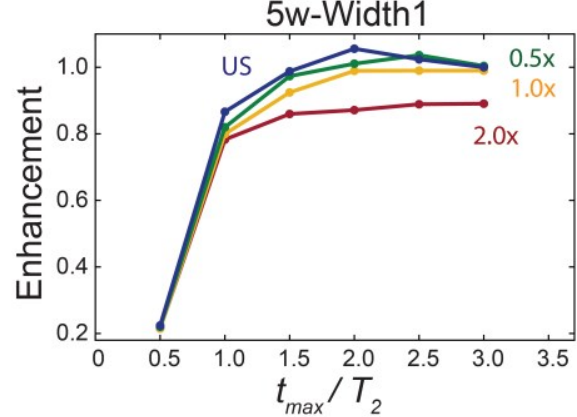

5w-Width2

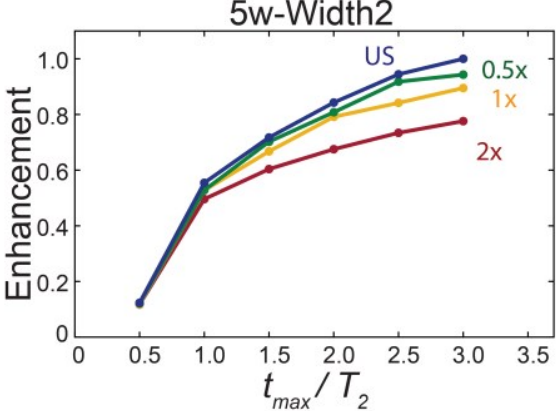

Figure S4 (continued): NUS knowledge enhancements in peak amplitude (area), frequency offset (position), and linewidth for two peaks separated by (c) 2 or (d) 5 times the linewidth under the indicated NUS biases. The peaks have equal amplitudes, but the second peak has a linewidth half that of the first. Both the separation and the NUS biases are defined with respect to the linewidth of peak 1 . For each simulation, the total number of time-domain points sampled was held constant, corresponding to equivalent total experiment times. In all cases, the intrinsic spectral knowledge was extracted from the time-domain FID's; reference spectra were generated from US to $3 T_{2}$. 

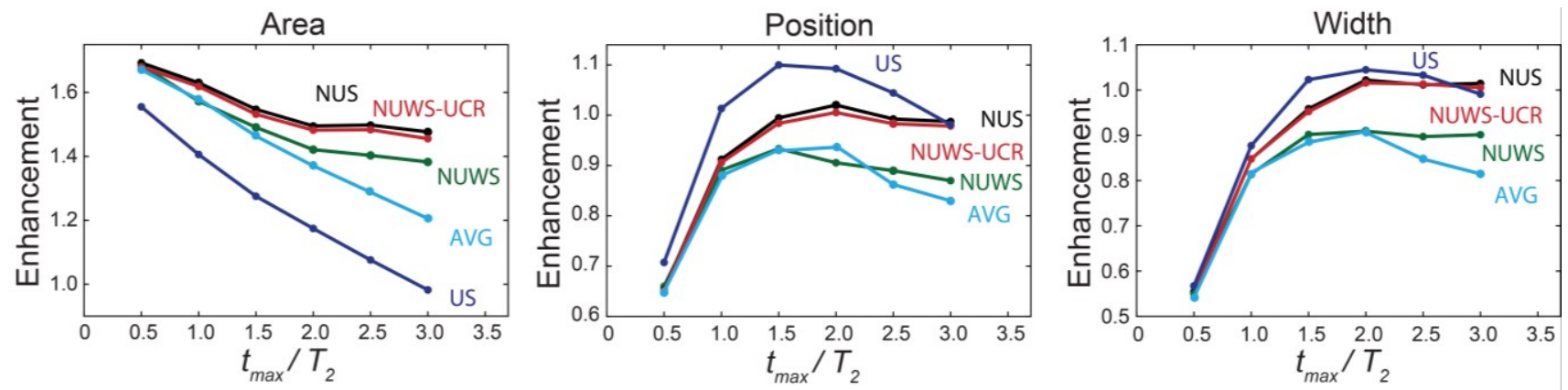

Figure S5: NUS knowledge enhancements in the frequency domain for spectra calculated with NUWS, NUWS-UCR, and the average (apodization-weighted) FID (AVG) for a single peak under matched NUS. For each simulation, the total number of time-domain points sampled was held constant, corresponding to equivalent total experiment times. The spectral knowledge was extracted from the frequency-domain spectra for NUWS, NUWS-UCR, and AVG; for comparison, the intrinsic spectral knowledge was extracted from the time-domain FID's for NUS and US. The enhancements for NUWS-UCR fully track those for NUS in the time domain. 

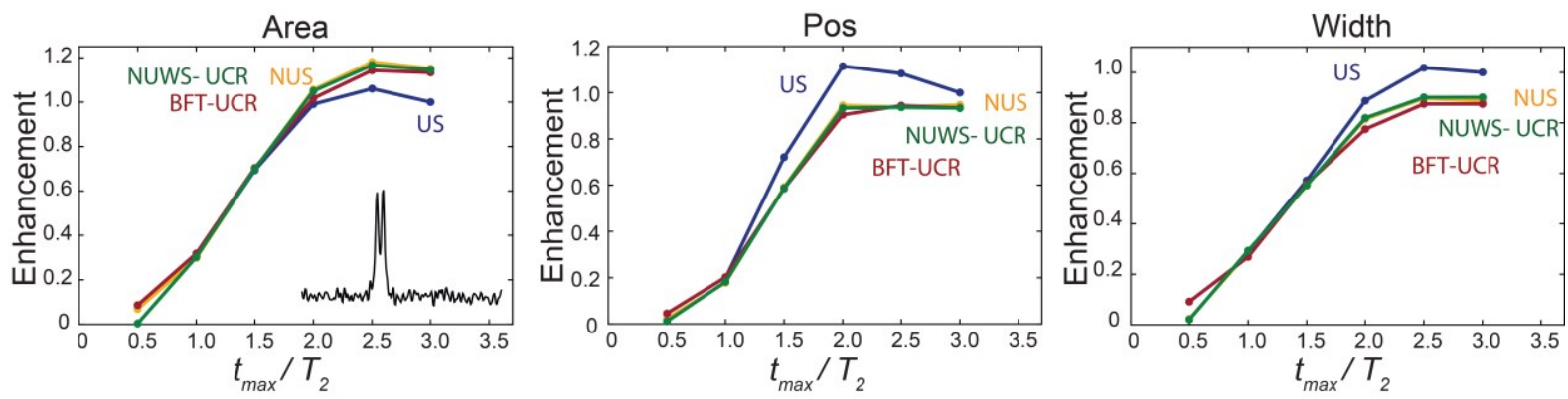

Figure S6: NUS knowledge enhancements in the frequency domain for spectra calculated with NUWS-UCR and BFT-UCR for two equal amplitude and linewidth peaks separated by 2 times the linewidth under matched NUS. For each simulation, the total number of time-domain points sampled was held constant, corresponding to equivalent total experiment times. The spectral knowledge was extracted from the frequency-domain spectra for NUWS-UCR and BFT-UCR; for comparison, the intrinsic spectral knowledge was extracted from the time-domain FID's for NUS and US. The spectral knowledge of peak amplitude (area), frequency offset (position), and linewidth exactly track those for NUS in the time-domain. 

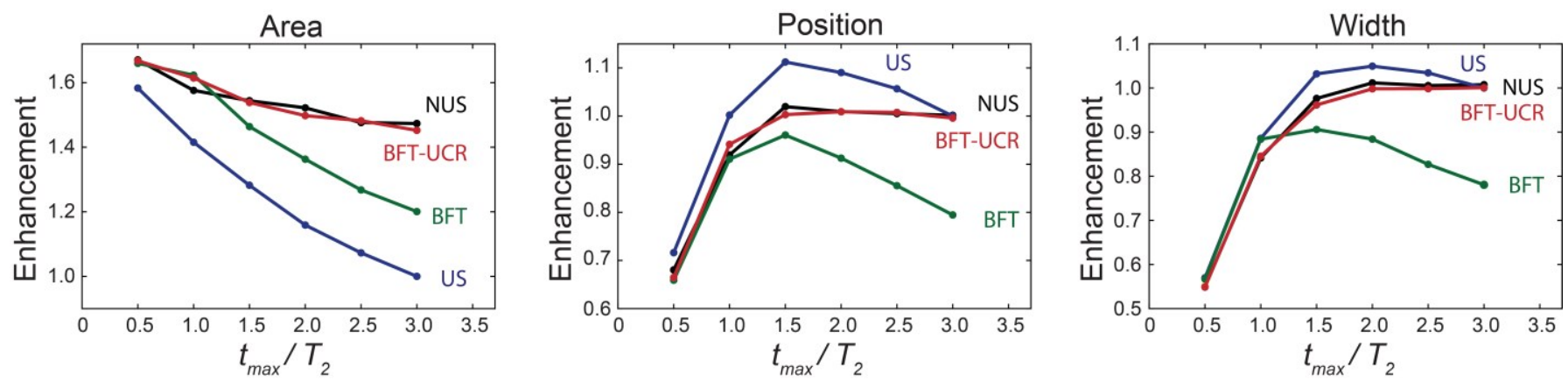

Figure S7: NUS knowledge enhancements in the frequency domain for spectra calculated with the BFT and the BFT-UCR for a single peak under matched NUS. For each simulation, the total number of timedomain points sampled was held constant, corresponding to equivalent total experiment times. The spectral knowledge was extracted from the frequency-domain spectra for BFT and BFT-UCR; for comparison, the intrinsic spectral knowledge was extracted from the time-domain FID's for NUS and US. The enhancements for BFT-UCR fully track those for NUS in the time domain. 